\title{
Volume Preserving Simulation of Soft Tissue with Skin
}

\author{
SEUNG HEON SHEEN, University of British Columbia, Canada \\ EGOR LARIONOV, University of British Columbia, Canada \\ DINESH K. PAI, University of British Columbia, Canada and Vital Mechanics Research, Canada
}
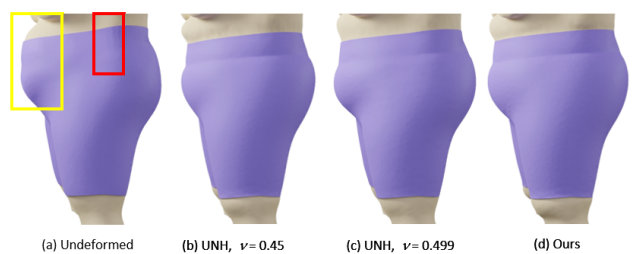

(d) Ours
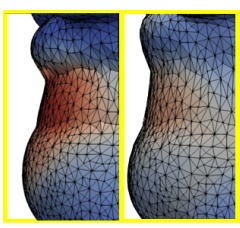

(e) $\mathrm{UNH}, v=0.45 \quad$ (f) $\mathrm{UNH}, v=0.499$
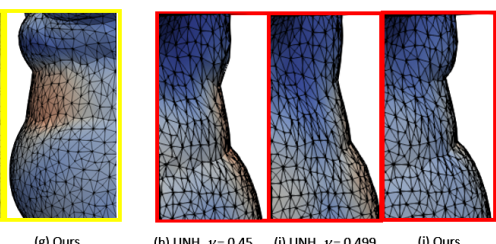

(g) Ours

Fig. 1. Simulating the fit of a tight garment requires realistic soft tissue deformation and illustrates the challenges of Finite Element Methods for human body simulations. Human tissues are soft but nearly incompressible. The neo-Hookean models popular in computer graphics enforce incompressibility indirectly, using a Poisson's ratio $v$ approaching 0.5 ; we call this standard approach "Unconstrained neo-Hookean" (UNH) Naively using $v=0.4545$ produces qualitatively reasonable deformation, but the body loses a significant amount ( 19\%) of its volume, mostly in regions of high compression (see (b), and close-ups (e,h)). Using $v=0.499$ preserves body volume up to an error of $0.5 \%$, but produces limited deformations due to locking $(\mathrm{c}, \mathrm{f}, \mathrm{i})$. By contrast, in our "Constrained neo-Hookean" $(\mathrm{CNH})$ method, we can designate volume preserving zones to match anatomical compartments, and exactly conserve volume within each zone while avoiding problems with volumetric locking. This results in more realistic displacement of soft tissues (d,g,j).

In addition to visual differences, volume preservation can lead to significant differences in the predictions of how well the garment fits; UNH with $v=0.4545$ predicts a waistband circumference $7 \mathrm{~cm}$ smaller than that with $v=0.499$, and $4.5 \mathrm{~cm}$ smaller than with our method. Our results can improve predictions of human soft tissue mechanics in applications ranging from virtual try-ons to visual effects.

Simulation of human soft tissues in contact with their environment is essential in many fields, including visual effects and apparel design. Biological tissues are nearly incompressible. However, standard methods employ compressible elasticity models and achieve incompressibility indirectly by setting Poisson's ratio to be close to 0.5 . This approach can produce results that are plausible qualitatively but inaccurate quantatively. This approach also causes numerical instabilities and locking in coarse discretizations or otherwise poses a prohibitive restriction on the size of the time step. We propose a novel approach to alleviate these issues by replacing indirect volume preservation using Poisson's ratios with direct enforcement of zonal volume constraints, while controlling fine-scale volumetric deformation through a cell-wise compression penalty. To increase realism, we propose an epidermis model to mimic the dramatically higher surface stiffness on real skinned bodies. We demonstrate that our method produces stable realistic deformations with precise volume preservation but without locking artifacts. Due to the volume preservation not being tied to mesh discretization, our method also allows a resolution consistent simulation of incompressible materials. Our

Authors' addresses: Seung Heon Sheen, shsheen@cs.ubc.ca, University of British Columbia, Vancouver, British Columbia, Canada; Egor Larionov, egor@cs.ubc.ca, University of British Columbia, Vancouver, British Columbia, Canada; Dinesh K. Pai, pai@cs.ubc.ca, University of British Columbia, Computer Science, Vancouver, BC, Canada and Vital Mechanics Research, Vancouver, BC, Canada.

Permission to make digital or hard copies of part or all of this work for personal or classroom use is granted without fee provided that copies are not made or distributed for profit or commercial advantage and that copies bear this notice and the full citation on the first page. Copyrights for third-party components of this work must be honored. For all other uses, contact the owner/author(s).

(C) 2021 Copyright held by the owner/author(s).

2577-6193/2021/9-ART

https://doi.org/10.1145/3480143

Proc. ACM Comput. Graph. Interact. Tech., Vol. 4, No. 3, Article . Publication date: September 2021. 
method improves the stability of the standard neo-Hookean model and the general compression recovery in the Stable neo-Hookean model.

CCS Concepts: • Computing methodologies $\rightarrow$ Physical simulation.

Additional Key Words and Phrases: volume constraint, incompressibility, finite element method, soft-tissue simulation, neo-Hookean elasticity

\section{ACM Reference Format:}

Seung Heon Sheen, Egor Larionov, and Dinesh K. Pai. 2021. Volume Preserving Simulation of Soft Tissue with Skin. Proc. ACM Comput. Graph. Interact. Tech. 4, 3 (September 2021), 23 pages. https://doi.org/10.1145/3480143

\section{INTRODUCTION}

Elastic materials are ubiquitous in everyday life. Many objects we interact with are organic in nature such as plants, animals, food, and most importantly our own bodies. Interestingly, most organic solids are nearly incompressible (due to their high water content), which makes them particularly difficult to simulate. Human soft tissue, for instance, is essentially incompressible, with a Poisson's ratio close to 0.5 [Fung 2013]. As a result, much of contemporary research in computer graphics focuses on robust simulation of incompressible hyperelastic solids (see Section 2). We focus on the popular neo-Hookean models, which are relatively simple while including non-linearity and the temptation to control incompressibility by setting Poisson's ratio $v \simeq 0.5$.

However, it is impossible to emulate true incompressibility and extremely difficult to simulate even near-incompressibility with this approach. This is because as the material approaches incompressibility $v \rightarrow 0.5$, the first Lamé parameter $\lambda \rightarrow+\infty$ (see Section 3 for background). The numerical and visual artifacts arising from the failure to correctly enforce incompressibility is known as volumetric locking (for ease of discussion, we will simply refer to it as 'locking'.)

There are multiple approaches to tackle this issue: the simplest being just using higher-order elements [Longva et al. 2020] or hexahedral elements. However, the increased computational cost and difficulty of implementation might not be desirable. Another class of popular methods is non-conforming finite elements (such as the Discontinous Galerkin class of methods), where the additional or non-conforming degrees of freedom allow significant deformation and therefore reduce the stiffness of the system. The last approach includes methods that seek to remove these element-wise constraints through Mixed Finite Elements or coarsened constraints, both of which are related to our method.

Our core idea is to tease apart the concept of incompressibility, a constraint on a derivative (the deformation gradient) from the related concept of volume preservation, a constraint on an integral (the volume of a finite region of material that we call a "zone"). Incompressibility is enforced per element in the standard neo-Hookean models, usually implicitly, using an energy term. By contrast, we enforce volume preservation as an explicit constraint on the volume of a zone. Volume preservation gives us considerable flexibility to choose larger zones that span multiple elements, zones that are independent of discretization, and zones that are aligned with meaningful anatomical tissue compartments (muscles, abdomen, breast, etc.). Zones may also overlap (e.g., we can preserve both the total volume of a body, and volumes of important tissue compartments).

A second key idea is that since volume preservation is already enforced using constraints, we can use much smaller values of $\lambda$ or $v$, thereby avoiding locking and related numerical instabilities. This can, of course, lead to volume loss per element but that will be compensated by volume gain in other elements in a zone to preserve volume. In other words, our simulation mesh may be viewed as a type of Arbitrary Lagrangian-Eulerian (ALE) mesh, in which volume is never lost but allowed to flow from one cell to another. Locking is always aggravated when using a coarse simulation mesh, but our method allows a stable simulation of volume preserving materials with a coarse 


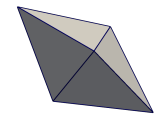

(a)

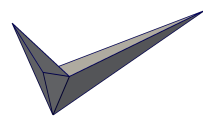

(b)

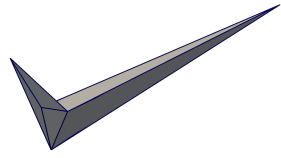

(c)

Fig. 2. Two Tet Simulation: (a) The reference state of the mesh, where two tetrahedra of equal volume are joined together by a face. The left tetrahedron is constrained by Dirichlet boundary conditions to be compressed into a plane. (b) With an unconstrained neo-Hookean (UNH) model Without the volume constraint, the total volume of the final mesh is $46.628 \%$ of the original. (c) With a volume constraint $(\mathrm{CNH}$ model), the tetrahedron on the right inflates to twice the volume to keep the total volume constant.

mesh. To our knowledge, this technique has not yet been closely studied with FEM simulations in computer graphics.

Note that $\lambda$ now controls only element volume, rather than the incompressibility of the material. In the rest of the paper we will repurpose the first Lamé parameter $\lambda$ to control volume change per element, instead of zonal volume change. This allows us to penalize extreme volume loss per element. We address this in Section 5 with an amendment to the volume penalty term found in compressible elastic energy models. Additionally, the proposed correction improves the compression response when using invertible energies.

Human bodies are covered by a layer of skin, a complex multi-layered structure. The outer layer comprising the epidermis is much stiffer than the underlying tissues, and significantly affects the quality of deformation. We propose a simple model of the epidermis and show that this extension contributes heavily to the appearance of realistic tissue deformation.

A simple illustration of these ideas is given in Figure 2. It illustrates the more general scenario in which locking artifacts increase at lower mesh resolutions, whereas our volume preservation is independent of the discretization of the zones.

Figure 1 shows the practical relevance of good volume preservation. Closeups of the belly (yellow boxes) and side waist in front view (red boxes) depict tissue displacement in false color, and yield more insights. We see that the traditional Unconstrained neo-Hookean (UNH) model compresses under the waistband by losing volume, without significantly extruding tissue away (e,h), whereas our method extrudes tissue more realistically, producing a sharp bulge $(\mathrm{g}, \mathrm{j})$ due to volume preservation. Increasing $v$ doesn't help the UNH models since locking reduces the deformation (f,i)

Contributions: we propose a new approach for simulating human tissues and other soft objects that preserve volume, while avoiding the common pitfalls of standard incompressible elasticity models. In addition to avoiding locking artifacts, our zonal volume constraint formulation makes deformation independent of discretization, and allows zones to be aligned with meaningful anatomical tissue compartments. In addition, we repurpose the first Lamé parameter to support inversion robustness and introduce a new form of the local compression penalty. We also extend the elastic energy potential to model the stiff epidermis, and demonstrate its importance. Finally, we propose a simple but complete pipeline for assigning volumetric zones using weights on the surface of the volumetric mesh, and demonstrate the application of these methods to predicting the fit of tight fitting garments.

\section{RELATED WORK}

A number of recent contributions have significantly improved the performance and behavior of hyperelastic solid simulations. The standard approach is using the Finite Element Method (FEM) on a Lagrangian tetrahedral (or hexahedral) mesh [Sifakis and Barbic 2012]. The methods used for 
soft tissue simulations are typically split between linear and non-linear hyperelasticity models. A number of popular elasticity models are used for soft tissue simulation including neo-Hookean, St. Venant-Kirchhoff, and co-rotated elasticity.

Co-rotated elasticity [McAdams et al. 2011; Müller et al. 2002], has been tremendously successful in real-time and interactive applications largely due to its simplicity. However, it suffers from element degeneration in large deformations [Civit-Flores and Susín 2014] and has poor volume preservation properties [Smith et al. 2018]. Non-linear energy models like the neo-Hookean models [Bonet and Wood 2008], have been used to circumvent these issues at a larger computational cost; although in recent years, neo-Hookean elasticity has also appeared in interactive simulations [Liu et al. 2017].

Our work targets invariant-based non-linear hyperelastic models, such as neo-Hookean elasticity, for their generality, superb handling of large deformations and inherent reflection stability.

Among non-linear elastic models are compressible and incompressible hyperelastic models. While incompressible models [Mooney 1940; Rivlin and Rideal 1948] pose an explicit volume constraint on each element, compressible models prevent severe compression using a penalty term [Bonet and Wood 2008]. The most popular method for solving elasticity problems in computer graphics is the standard linear FEM on a Lagrangian mesh because of its performance profile and versatility. Unfortunately, imposing a severe penalty - let alone a hard constraint - for volume change on each element can cause severe numerical difficulties and locking, especially in linear tetrahedral FEM.

In traditional FEM, volumetric locking is addressed by decoupling incompressibility from displacements with Mixed Finite Element Methods. In computer graphics, Irving et al. [2007] have addressed locking in tetrahedral meshes by softly constraining the volume of the one ring around each vertex in a tetrahedral mesh using position and velocity correction steps. This approach is an application of nodal strain elements [Bonet and Burton 1998], where stress and strain, in this case their volumetric component, is nodally interpolated in a mixed FEM. However, without additional stabilization of some sort, these types of mixed elements are known to be unstable and the number of additional pressure variables are proportional to the number of nodes. A further discussion of Mixed Finite Element Methods is presented in the next section. Kaufmann et al. [2012] looked to solve locking by introducing additional degrees of freedom to the system by using a Discontinuous Galerkin discretization. Smith et al. [2018] proposed the Stable neo-Hookean energy model to handle invertible elements as well as improve stability for high Poisson's ratios.

Irving et al. [2007] proposed a method of (weakly) constraining volume in each 1-ring neighborhood of every mesh vertex. This method is an implementation of the Average Nodal Pressure element [Bonet and Burton 1998], where the cell-wise constant pressure samples of the one-ring neighbors are averaged on the nodes. By contrast, our method uses pressure samples (i.e. the volume preserving zones) that are coarser and decoupled from the mesh topology. Fine-scale cell-wise pressures are instead controlled through a local penalty, which avoids additional pressure variables. This way we can keep $\lambda$ low enough to avoid locking, while simultaneously enforcing volume preservation. Our method has significantly fewer constraints, compared to the total number of vertices, which permits enforcing constraints exactly using constrained minimization to solve the variational problem rather than using constraint projection.

A recent work in computer graphics by Francu et al [2021] targets the problem of locking by using a linear-linear mixed formulation. However, as noted by the authors, this formulation does not satisfy the Babŭska-Brezzi inf-sup condition and thus will result in inaccurate pressure solutions. Specifically, their results show the same problems of checkerboard patterns in the pressure modes. Although the spurious modes do not affect the displacement results as the authors mention, it causes problems when a reasonable computation of pressures is necessary, for example when one

Proc. ACM Comput. Graph. Interact. Tech., Vol. 4, No. 3, Article . Publication date: September 2021. 
needs them to simulate frictional contact. Our method does not suffer from this issue, since we avoid the spurious modes in pressure by sampling pressures from a coarser scale compared to the displacements. Compare their Figure 15 to our Figure 7. Our method also requires fewer constraints compared to theirs, since we only require one constraint per "zone", while they require a constraint per node.

Some works have targeted global volume preservation [Diziol et al. 2011; Hirota et al. 2000; Hong et al. 2006; Promayon et al. 1996], however not in the context of volumetric FEM. Global volume constraints have also been applied in studies in skinning methods [Rohmer et al. 2009]. Some also proposed using a sweep-based approach to conserve the volume [Angelidis et al. 2004; Yoon and Kim 2006] or a vector field approach [von Funck et al. 2007]. By contrast, we propose zonal volume constraints for neo-Hookean type energy models for Lagrangian FEM simulations.

Finite element simulations also suffer from element inversions during severe deformation. Inversion stability allows simulations to handle large deformations and permits taking large time steps, which can improve simulation performance significantly. A line of recent work has proposed methods for resolving element inversions by extending the energy density function to the negative volume region. Force filtering methods [Irving et al. 2004; Teran et al. 2005] have improved inversion stability but suffer from subtle problems including invalid inversion recovery directions or derivative drift as thoroughly explored in [Smith et al. 2018]. Stomakhin et al. [2012] propose a $C^{1}$ or $C^{2}$ extension of the entire energy density function for low volume fractions, which resolves many of these problems. However, filtering methods can be quite sensitive to appropriate specification of filtering thresholds and reflection conventions [Wang and Yang 2016]. We instead follow a simpler approach similar to [Smith et al. 2018], where we design a volumetric penalty term to satisfy necessary conditions for stability and inversion robustness. Our penalty function improves upon the Stable neo-Hookean volumetric term by also introducing nonlinearity to the stress, resulting in better inversion recovery and improved performance.

\section{BACKGROUND}

\subsection{Variational Elasticity}

In this section, we establish the context for our contributions by introducing FEM simulation of hyperelastic materials as a variational problem.

Let $\Omega \subset \mathbb{R}^{3}$ be a union of mesh tetrahedra representing an elastic solid in its undeformed configuration. Then let $\mathbf{x} \in \mathbb{R}^{3 n}$ correspond to a stacked vector of mesh vertex positions that prescribe the deformation of the solid, where $n$ is the total number of vertices in the mesh. In an elasticity problem, we are interested in finding the configuration $\mathbf{x}$ that results in the lowest potential energy for the elastic solid $\Omega$ given a set of boundary conditions and external forces. Mathematically, we may write the problem statement as

$$
\mathbf{x}^{*}:=\underset{\mathbf{x}}{\arg \min } W(\mathbf{x})
$$

where $W(\mathbf{x})$ represents the elastic work function for configuration $\mathbf{x}$. This formulation allows conservative external forces to be added as additional potentials in the objective, however for the sake of simplicity we ignore external forces in the following sections.

With linear (constant strain) elements, $W$, which is the integral of energy density function $\Psi(\mathbf{x})$, can be written as the sum of volume-scaled per-element energies:

$$
W(\mathbf{x})=\int_{\Omega} \Psi(\mathbf{x}):=\sum_{e} V_{e} \Psi\left(\mathbf{F}_{e}(\mathbf{x})\right),
$$

Proc. ACM Comput. Graph. Interact. Tech., Vol. 4, No. 3, Article . Publication date: September 2021 
where $V_{e}$ is the volume of element $e$ in the reference configuration, which depends on the element deformation gradient $\mathbf{F}_{e}$. The choice of the energy density function $\Psi$ determines the hyperelastic energy model.

For the time discretization, we may use any integration method. In the dynamics examples below we adopt the implicit Euler time integrator and add an inertial energy term to this minimization. However for simplicity of exposition we focus on static FEM to describe our approach to volume preservation.

\subsection{Incompressibility and Locking}

There are two ways in which incompressibility could be enforced: either directly, as a constraint that the volume is preserved, or indirectly with a penalty term that powerfully resists compression. Since both ways are frequently referred to as "incompressible," to avoid confusion we will refer to incompressible neo-Hookean models using the first method as "Constrained neo-Hookean" $(\mathrm{CNH})$, and those using the second method as "Unconstrained neo-Hookean"(UNH).

Most incompressible hyperelastic energy models used in graphics are of the Unconstrained neo-Hookean type, and penalize element-wise volume change with a term scaled by the first Lamé parameter $\lambda$, which depends on the Young's Modulus $E$ and Poisson's Ratio $v$ as:

$$
\lambda=\frac{E v}{(1+v)(1-2 v)} \text {. }
$$

For instance, the most common version of such an energy density function [Bonet and Wood 2008] is written as

$$
\Psi_{\mathrm{UNH}}(\mathbf{F} ; \lambda, \mu)=\frac{\mu}{2}\left(I_{C}-3\right)-\mu \log J+\frac{\lambda}{2}(\log J)^{2}
$$

where $I_{C}=\operatorname{tr}\left(\mathbf{F}^{\top} \mathbf{F}\right)$, and $J=\operatorname{det}(\mathbf{F})$ represents the fraction of volume after deformation. This means that when $J$ is close to zero (extreme compression), $\Psi_{\mathrm{UNH}}$ will generate large penalty forces to restore the element to reference configuration. Another commonly used material model, co-rotated elasticity [McAdams et al. 2011] is written as

$$
\Psi_{\mathrm{CR}}(\mathbf{F} ; \lambda, \mu)=\mu\|\mathbf{F}-\mathbf{R}\|_{F}^{2}+\frac{\lambda}{2} \operatorname{tr}(\mathbf{S}-\mathbf{I})^{2},
$$

where $\mathbf{R}$ and $\mathbf{S}$ form the polar decomposition: $\mathbf{F}=\mathbf{R S}$ and $\mathbf{I}$ is the $3 \times 3$ identity matrix. Here, in a similar fashion, local compression is once again penalized by $\lambda$.

There are multiple aspects of locking which are problematic for simulating volume preserving elastic solids. First, high Poisson's ratios make the system stiff, which results in stiffness related issues such as instability and artificial damping. Somewhat related to this, when using linear tetrahedral elements and element-wise volume constraints, the resulting system becomes highly overconstrained. However, the main problem arises from the choice of the constitutive equation, when volumetric stress depends on $\lambda$. In classical FEM theory, Céa's Lemma dictates that the quasi-best approximation error depends not only on mesh discretization error, but also on $\lambda$. Hence, when $\lambda \rightarrow+\infty$, the finite element solution can no longer be a reliable predictor of the solution of the PDE. A more detailed explanation can be found in Braess [2007].

\subsection{Mixed Finite Element Methods}

It is often necessary to compute reliable solutions not only for displacements but also for pressures (e.g., for frictional contact or fractures). For displacement-based one-field FEM, pressure must be computed from the displacement variables $\mathbf{x}$. Specifically, cell-wise hydrostatic pressure is usually computed as the negative of the divergence of the Cauchy stress tensor. Since the Cauchy stress 
tensor is related to the derivative of the energy density function $\Psi(\mathbf{x})$, the pressures computed from a one-field FEM mainly depend on the volume term of $\Psi$. However, due to similar issues as discussed above, when the material is incompressible and $\lambda \rightarrow+\infty$ the volume term stops being a reliable model for volumetric stress.

One traditional way of decoupling incompressibility from $\lambda$ is by introducing an additional pressure variable $\mathbf{p}$ that models the volumetric stress component of elements, interpolated separately from displacement on the finite element mesh [Bathe 2006]. This allows us to reformulate the variational problem as

$$
\mathbf{x}^{*}:=\arg \max _{\mathbf{p}} \min _{\mathbf{x}} \int_{\Omega} \hat{\Psi}(\mathbf{x})+\mathbf{p}^{T} \mathbf{c}(\mathbf{x})
$$

where $\hat{\Psi}(\mathbf{x})$ is the deviatoric component of the displacement-based elastic potential, and $\mathbf{c}(\mathbf{x})$ is a term that relates $\mathbf{p}$ to $\mathbf{x}$. This additional term can be interpreted as a constraint on $\mathbf{p}$ to be proportional to the hydrostatic pressure computed from the displacements $\mathbf{x}$. Then, $\mathbf{p}$ becomes the Lagrange multiplier for the constraint $\mathbf{c}(\mathbf{x})$. One implementation of this type of formulation is shown in Sussman et al. [1987]. These methods are known as the displacement-pressure Mixed Finite Element Methods and are one of the most accurate ways to solve the problem.

With the additional degree of freedom, the Babŭska-Brezzi inf-sup condition restricts the choice of the space of finite element basis for the additional variable for the method to be stable [Bathe 2001]. This condition dictates that the order of basis for the displacement variables must be higher than that of the pressure variables. Specifically, for conforming tetrahedral elements the lowest order finite element space choices are either the Hood-Taylor elements $\left(P_{2}\right.$ for $\mathbf{x} / P_{1}$ for $\mathbf{p}$, where $P_{k}$ denotes the space of $k$-th order polynomials), or MINI $\left(P_{1}^{+} / P_{1}\right.$, where superscript + denotes an enrichment of cubic bubble [Arnold et al. 1984]). Hence, Mixed FEM with a simple linear tetrahedral Finite Element basis for displacement is usually not valid for stable simulations. This includes the Average Nodal Pressure elements proposed in Irving et al. [2007], where the Lagrange multipliers of 1-ring volume constraints can be interpreted as cell-wise constant pressure variables $\left(P_{0}\right)$ being averaged on the nodes. Although this alleviates some of the problems arising from each element being constrained, it still fails to meet the inf-sup condition and spurious modes may occur without additional stabilization [Puso and Solberg 2006].

The intuition for our approach from mixed FEM is that, to achieve an efficient and stable computation of the additional pressure variables, one must sample the pressure variables in a coarser scale compared to the displacement variables. Then, we are able to split the pressure computation into a coarser and finer scale, to control the coarse-scale pressures as separate pressure variables as Lagrange multipliers for volume constraints, as in Eq.5, and compute the fine-scale pressures from displacements. Therefore, we look to a much more efficient and simpler approach by enforcing a volume constraint for a few larger zones of elements, and modeling the element-wise local pressure as an additional local penalty term.

\section{ZONAL VOLUME CONSTRAINT}

To solve the problem of volumetric locking, instead of enforcing a per-tetrahedron near-incompressibility with high Poisson's ratio, we adopt the approach of the Mixed Finite Element Method. Essentially, we solve the saddle-point system as a constrained minimization with constraint function $\mathbf{c}(\mathbf{x})=\mathbf{0}$. Specifically, our constraint enforces the total volumes of zones defined as local sets of finite elements to be preserved. Compared to other mixed elements, our approach is much more efficient and easier to implement while showing comparable results. Moreover, our approach provides the modeling flexibility of choosing zones that are aligned with anatomical compartments (see Section 6). 
Each constraint is simply formulated as the requirement that the total volume of all elements in a specified zone of the deformed mesh is equal to the initial volume. That is, for $j$-th zone $G_{j}$, the zonal volume constraint function $c_{j}$ is defined as follows,

$$
c_{j}(\mathbf{x})=\sum_{e \in \zeta_{j}} V_{e}(\mathbf{x})-V_{e}^{0},
$$

where $V_{e}^{0}$ is the reference volume of element $e$, which belongs to zone $j$ with element index set $\zeta_{j}$.

Imposing this constraint for each zone gives us a new constrained minimization problem:

$$
\begin{aligned}
& \underset{\mathbf{x}}{\arg \min } \int_{\Omega} \Psi(\mathbf{x}) \\
& \text { s.t. } c_{j}(\mathbf{x})=0 \quad \forall j \text {. }
\end{aligned}
$$

As a special case we can preserve the total volume with a single global constraint; by contrast, classical incompressible neo-Hookean models require the volume of each and every element to be preserved.

To illustrate the simplicity of this type of constraint we define the volume constraint for a tetrahedral mesh. The volume of the tetrahedron $e$ is defined (up to a constant scaling) as the triple scalar product

$$
V_{e}(\mathbf{x})=\mathbf{v}_{3} \cdot\left(\mathbf{v}_{1} \times \mathbf{v}_{2}\right),
$$

where $\mathbf{v}_{i}=\mathbf{x}_{i}-\mathbf{x}_{0}$. Then the Jacobian of the constraint function can be computed as

$$
\frac{\partial c_{j}}{\partial \mathbf{x}}=\sum_{e} \frac{\partial V_{e}}{\partial \mathbf{x}},
$$

where the sparse vector $\frac{\partial V_{e}}{\partial \mathrm{x}} \in \mathbb{R}^{3 n}$ is zero everywhere except for the vertices of element $e$, where

$$
\begin{gathered}
{\left[\frac{\partial V_{e}}{\partial \mathbf{x}}\right]_{0}=-\left(\mathbf{v}_{2} \times \mathbf{v}_{3}+\mathbf{v}_{3} \times \mathbf{v}_{1}+\mathbf{v}_{1} \times \mathbf{v}_{2}\right)} \\
{\left[\frac{\partial V_{e}}{\partial \mathbf{x}}\right]_{1}=\mathbf{v}_{2} \times \mathbf{v}_{3}, \quad\left[\frac{\partial V_{e}}{\partial \mathbf{x}}\right]_{2}=\mathbf{v}_{3} \times \mathbf{v}_{1}, \quad\left[\frac{\partial V_{e}}{\partial \mathbf{x}}\right]_{3}=\mathbf{v}_{1} \times \mathbf{v}_{2} .}
\end{gathered}
$$

Finally, the Hessian stencils for each $V_{e}$ will be simple linear skew-symmetric matrices. Thus, $c_{j}(\mathbf{x})=0$ is a one dimensional constraint with simple to implement sparse derivatives, which gives true volume preservation. Note that this constraint can be further optimized by computing the volume of the entire zone by iterating over zone boundary faces only.

Although uncomplicated, this constraint provides a powerful tool for emulating incompressible elasticity. It allows users to achieve volume preservation without increasing Poisson's ratio, which can cause instabilities and locking. The constrained optimization problem can be solved with any non-linear optimizer which can deal with nonlinear constraints. For most nonlinear solvers a few equality constraints should not be prohibitively expensive to solve, but for additional performance gain one may naturally use an Augmented Lagrangian method to solve the constraints.

The zone sizes are important in determining the level of local incompressibility. One global zone for the entire mesh will essentially be a hydrostatic simulation, analogous to simulating a water balloon. As the zone sizes decrease, there will be more local incompressibility around each element which will result in a stiffer behavior. However, as long as the zones are at least as large as the 1-ring [Irving et al. 2007], volumetric locking will not occur. Therefore, as we use a smaller zone sizing, the results will become more similar to the results in [Irving et al. 2007], but at a steeper performance cost. 
Our method can be viewed as a simplification of the 2-field mixed formulation, where the pressure potential is given as

$$
\mathbf{p}^{T} \mathbf{c}(\mathbf{x})=\sum_{j} \mathbf{p}_{j} c_{j}(\mathbf{x})=\sum_{j} \mathbf{p}_{j}\left(\sum_{e \in G_{j}} V_{e}(\mathbf{x})-V_{e}^{0}\right),
$$

where the interpolated hydrostatic pressures $\mathbf{p}_{j}$ for zone $j$ are identified to be the Lagrange multipliers for the $j$-th zonal volume constraint. If each element was assigned to a unique zone, our method would recreate the mixed-element formulation for incompressible materials. However, we use only a handful of zones, which keeps the problem size small and avoids locking and instability.

\subsection{Stabilization}

We apply the F-bar method [de Souza Neto et al. 2005] to the energy density function to ensure stability. This method relaxes the near-incompressibility constraint enforced by the constitutive model by a modification of the deformation gradient, based on a multiplicative split of the deformation gradient $\mathbf{F}$ into a deviatoric and volumetric component. The deviatoric part is then computed as

$$
\overline{\mathbf{F}}=\alpha \mathbf{F}, \quad \text { where } \alpha=\frac{\bar{J}^{\frac{1}{3}}}{J^{\frac{1}{3}}},
$$

and $\bar{J}$ is the average of $J$ computed over a set of local element stencils. In our case, the local sets are the zones where the total volume is preserved, hence conveniently $\bar{J}=1$, and $\alpha=J^{-\frac{1}{3}}$. However, since as $J \rightarrow 0$ we have that $\alpha \rightarrow+$ inf, we instead apply a $C^{2}$ extension to $\alpha$ below a certain threshold $\epsilon$, similarly to Stomakhin et al. [2012]. Then the new extended deviatoric projector is given as

$$
\tilde{\alpha}:= \begin{cases}J^{-\frac{1}{3}} & \text { for } J>\epsilon \\ \epsilon^{-\frac{1}{3}}-\frac{1}{3} \epsilon^{-\frac{4}{3}}(J-\epsilon)+\frac{2}{9} \epsilon^{-\frac{7}{3}}(J-\epsilon)^{2} & \text { for } J \leq \epsilon\end{cases}
$$

In practice, the choice of $\epsilon$ is not too important as long as it is small $(\sim 0.1)$.

We then use $\overline{\mathbf{F}}(\mathbf{x})$ to compute the deviatoric part of the constitutive equation. For example, the deviatoric part of neo-Hookean energy density function (4) will now be computed as

$$
\bar{\Psi}_{\mathrm{NH}}(\overline{\mathbf{F}} ; \lambda, \mu)=\frac{\mu}{2}\left(\tilde{\alpha}^{2} I_{C}-3\right) \text {. }
$$

Note that this is similar to the form presented by Rivlin [1948], but extended below $\epsilon$ to be continuously defined for $J \leq \epsilon$.

Using only the deviatoric component of deformation gradient for the elastic potential energy, we remove the contribution of the constitutive equation on the pressure. Hence, this allows the complete split of the total elastic stress, to the deviatoric stress from the elastic potential, and the volumetric stress from the constraint Lagrange multipliers and the volume penalty.

\section{LOCAL COMPRESSION PENALTY}

Our method ensures that volume is preserved within each zone, but without any element-wise compression penalty the volume inside each zone can transfer between elements. This is a feature, as discussed in the Introduction, since it reduces the cost and numerical challenges of enforcing volume preservation locally, while ensuring good behavior globally. Note that, unlike in the hydrostatic case, volume can not transfer completely freely since elastic forces due to the shear modulus restrict large flows.

However, the zonal constraints by themselves model only the hydrostatic pressure in the coarse zones, hence we also need to model the finer-scale pressures in the individual elements. We employ 
a more traditional approach to modeling element-wise pressure in the penalty method. To model this local compression penalty function, we look at the volume penalty functions present in various neo-Hookean elasticity models. In neo-Hookean models, the bulk modulus controls how much the material resists element-wise volumetric deformation. The bulk modulus is represented in most neo-Hookean energy formulations in the first Lamé parameter $\lambda$, which is a combination of the shear and bulk modulus. However, as discussed in Section 3, when $\lambda \rightarrow+\infty$ locking occurs, and the pressure computations become unstable. But since we model the coarse-scale pressures as constraints, and we only need to model the finer-scale deviations in pressures, we are able to use a lower $\lambda$ and avoid locking.

If $\lambda$ is set too low the simulation is more susceptible to collapsing elements and even equilibrium configurations with inverted elements for invertible energy models. Consider the example in Figure 9 of a cylindrical puck with a moving Dirichlet boundary condition on a set of vertices on top of the puck. As the puck compresses, the tetrahedra underneath the moving boundary are flattened to the point where subsequent steps cause boundary-adjacent tetrahedra to invert. At this point, incompressible energy models with a logarithmic volume penalty $(\log J)$ term will become undefined because $J \leq 0$. Other models, like co-rotated elasticity, may permit inverted elements, but won't be able to recover from an inverted configuration. This issue has motivated a number of solutions [Irving et al. 2004; Smith et al. 2018] for handling inverted elements, but we will focus on the recent work on the Stable neo-Hookean model developed by Smith et al. While the proposed model attempts to solve many of the issues with non-invertible energies and doesn't require additional parameters, as can be seen from the plot of the volume change penalty term in terms of relative volume change $J$ in Figure 3, the Stable neo-Hookean energy resists compression much more timidly as compared to the standard neo-Hookean model defined in Equation 4.

This results in the simulation possibly converging to an invalid configuration where inverted elements exist, and a nonlinear optimization solver can struggle due to inverted elements being present in intermediate solutions which cause oscillations. Especially, this oscillation can be aggravated when constraints are introduced, presenting major performance issues when one tries to use volume constraints. We solve this by formulating a new volume penalty term that is both invertible and still resists compression effectively.

Let us write such penalty term as $U(J)$, controlled linearly by parameter $\lambda$. To design such a penalty term, we first take a look at what conditions the function $U(J)$ must meet. A detailed study of various neo-Hookean compression penalty terms and explanations for each of the conditions can be found in [Hartmann and Neff 2003].

a) The function must evaluate to 0 at rest $(J=1)$.

b) The gradient of the function, i.e., the volumetric stress, must also evaluate to 0 at rest.

c) For the $\lambda$ of the penalty term to correspond to the Lamé parameter in linear elasticity, $\frac{\partial^{2} U(1)}{\partial J^{2}}=1$ must hold.

d) The function must be defined for all real numbers $(-\infty,+\infty)$.

e) $\frac{\partial^{2} U(J)}{\partial J^{2}} \geq 0, \forall J \in \mathbb{R}$ for the penalty to both penalize compression and stretch.

Consider the following function,

$$
U(J ; \beta):=\frac{1}{12}(J-1)^{2}\left[\beta(J-1)^{2}+6\right]
$$




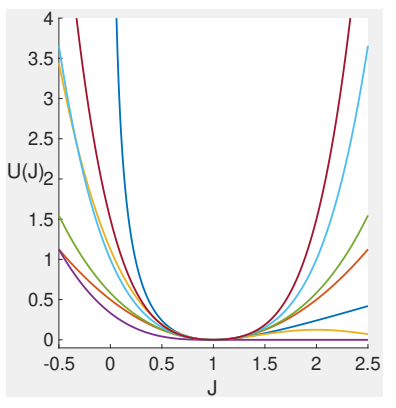

(a)

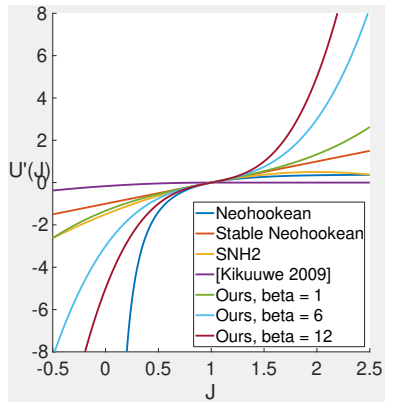

(b)

Fig. 3. Penalty Plots: A plot of the penalty terms $U(J)$ and their stresses $U^{\prime}(J)$ from different energy formulations (neo-Hookean, Stable neo-Hookean [Smith et al. 2018], the second-order expanded version of Stable neo-hookean (SNH2), [Kikuuwe et al. 2009], ours with $\beta=1$, and ours with $\beta=6$ ) with $\lambda=1$, in terms of the relative volume change $J$. The neo-Hookean volume term (blue) indicates a substantially larger penalty when compared to Stable neo-Hookean (orange) and [Kikuuwe et al. 2009] (yellow), but the penalty term is undefined when $J \leq 0$ due to its log term. This is more evident to see in the stress plot during compression $(J<1)$, where the Stable neo-Hookean volumetric stress changes in a linear manner. Our method for both $\beta=1$ (purple) and $\beta=6$ (green) shows much more effective penalization under compression and stretch. Our stresses shows a nonlinear dependence on $J$ similar to the neo-Hookean term, but also shows a effective growth not only during compression but also during stretch.

where the parameter $\beta \in[0,+\infty)$ controls how steeply the penalty function will penalize change in $J$. The first and second derivatives of the function are

$$
\begin{aligned}
& \frac{\partial U(J ; \beta)}{\partial J}=\frac{1}{3}(J-1)\left[\beta(J-1)^{2}+3\right], \text { and } \\
& \frac{\partial^{2} U(J ; \beta)}{\partial J^{2}}=\beta(J-1)^{2}+1 .
\end{aligned}
$$

Therefore, the function satisfies all of the conditions listed above. Note that for $\beta=0, U(J ; 0)=$ $U_{\mathrm{SNH}}(J)=\frac{1}{2}(J-1)^{2}$. As $\beta$ increases, the penalty function penalizes compression and stretch more effectively than the Stable neo-Hookean penalty term, while still being fully invertible. Therefore, this is a suitable choice for our compression penalty term. Plots comparing different penalty terms $U(J)$ and stresses $\frac{\partial U(J)}{\partial J}$ are shown in Figure 3. Experimentally, $\beta=1$ was sufficient for most realistic examples governed by external force, but for examples where inversions were more likely due to contact or boundary conditions, higher values of $\beta$ resolved all inversions.

The additional nonlinearity introduced in the gradient (Equation 15) of our penalty function compared to a standard Stable neo-Hookean penalty is the main reason for the inversion-robustness in our model. It is possible to formulate models with even higher nonlinearity than what we propose here, but in our experiments we found that such energy models provide no significant benefit in resolving inversions compared to (13) and only increase the number of nonlinear solver iterations until convergence. The plot in Figure 3 demonstrates how effectively our penalty resists compression compared to other invertible methods.

We demonstrate that by this simple addition to the energy potential, we can obtain results similar to that of using mixed finite elements as in [Irving et al. 2007], but with very few global constraints (or even one constraint). The results of Figure 7 demonstrate that with a local compression penalty equivalent to $v=0.45$ and with just one global zone, the deformations are close to using a 1-ring 
constraint around each vertex. Also, we found that simply adding this additional nonlinearity to the energy resulted in faster performances in most examples when volume constraints were used, and even in many cases where there were no constraints. In Table 1, we compare the performance results of using $\beta=0$ (equivalent to $\mathrm{SNH}$ ) and higher $\beta$.

\section{VOLUMETRIC ZONING}

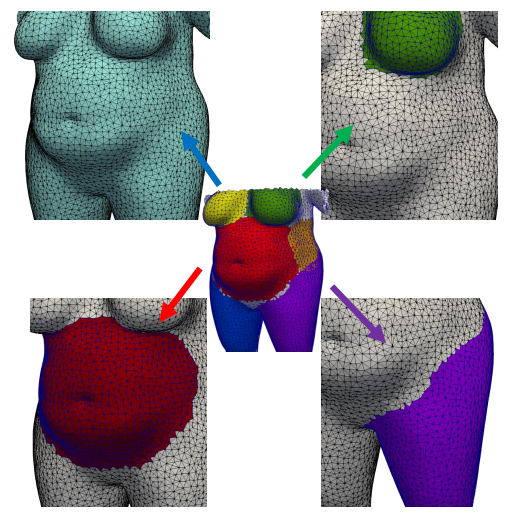

Fig. 4. Body Zones: Volume preserving zones on the body mesh used for simulation in Figure 1. Six significant anatomical compartments (belly (red), side and back waist (orange), 2 legs (blue and purple), 2 breasts (yellow and green)) are selected as zones. Additionally, another zone is added including the full body (cyan), so that the entire body would be volume preserving. Note that the full body zone is not necessary, but can be added to ensure the volume preservation of not only each compartments but also the entire body. The zones are drawn on the mesh surface with a texture painting tool in a visual effects software, and then projected to the inner body using our projection method.

Human soft tissues are not homogenous but naturally segmented into anatomical compartments. Our zonal volume preservation method provides additional flexibility for modelers to align volume preservation with such anatomical compartments (see Figure 4 for example). For a more complete description of one possible workflow for defining such zones, we propose a simple method of obtaining volumetric zones on a tetrahedral mesh from surface vertex annotation.

Manual vertex painting is a very common part of many character animation pipelines, allowing users to assign attributes such as skinning weights. Alternatively, there are methods to automatically compute transformation weights from skeleton meshes [Baran and Popović 2007; Rohmer et al. 2009; Weber et al. 2007], from sparse subsets of degrees of freedom [Jacobson et al. 2012], or from animation data [James and Twigg 2005].

Using either of the aforementioned methods, we end up with a set of weights defining possibly overlapping zones on the surface of our FEM mesh. We then transfer this data onto the surface triangles. Naturally these surface zones should be simply connected in order for the volumetric zones to follow suit.

In order to transfer zone information to the rest of the tetrahedral mesh, we first construct a smooth potential field around the mesh surface using Hermite Radial Basis Functions [Macêdo et al. 2009; Pai et al. 2018; Vaillant et al. 2013; Wendland 2004], although any signed distance field will do. We then project each tetrahedron centroid along the potential gradient onto the surface triangles. The triangle zone information is then copied from the triangles, back to the source tetrahedra. 


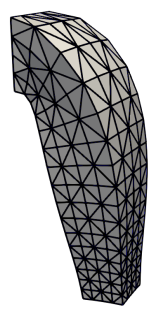

(a) Hydrostatic

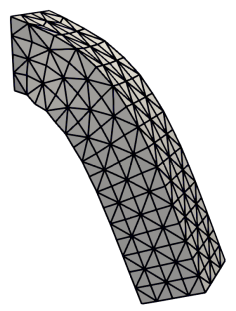

(d) 1-ring

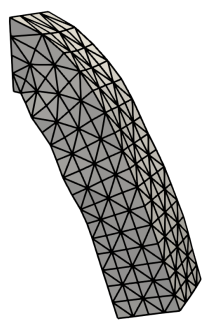

(b) 4-ring

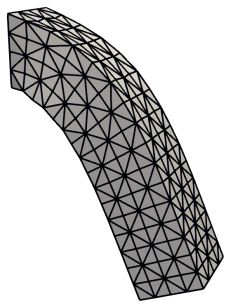

(e) Hydrostatic + Penalty

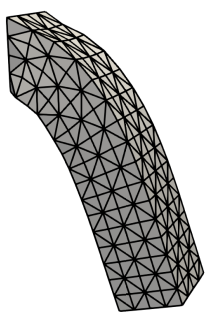

(c) 2-ring

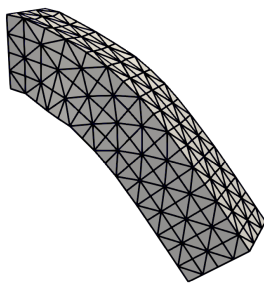

(f) $v=0.499$

Fig. 5. Zoning Tests: A cantilever beam with $16 \times 4 \times 4$ vertices is suspended under gravity with the left surface fixed with Dirichlet boundary conditions, ( $a, b, c, d)$ demonstrates the results of using different choices of volumetric zones without any extra penalty. (a) is the hydrostatic case, where the entire mesh is one zone; (b, c, d) respectively are the results of using a 4-ring, 2-ring, 1-ring zone [Irving et al. 2007]. (e) shows the result of our method with the hydrostatic zone with penalty, which closely resembles the 1-ring result. (f) is the case where a high Poisson's ratio was used, which is essentially a zone per element, where locking prevents the beam from bending further.

Albeit simple, this projection is an effective method to map internal tetrahedral mesh elements to surface triangles. This way, we allow the users to define volume preserving zones by simply painting surface vertices with any existing tool. We demonstrate this approach with an sample female body simulation mesh on Figure 4, where the surface zones are chosen manually to capture the anatomical volume-preserving regions.

The sizes of the volumetric zones determine the extent of the volume flow: smaller zones mean the volume flow is more restricted. This leads to the object appearing stiffer as the zones get smaller, and when each zone is a single element locking would occur. As the zones become smaller, the zonal volume constraints approximate the element-wise volume constraint more closely. Therefore the solution becomes more accurate, so long as stiffness is sufficiently low (see Section 3.2). However, volumetric stiffness inside zones can also be controlled through the penalty, which approximates the fine-scale pressures. This allows the user to use a much coarser choice of zones, with additional volume penalty to get an accurate result similar to using finer zones. Figure 5 demonstrates the results of a simple simulation of a cantilever beam, where different sized volumetric zones were used. Our method with a hydrostatic zone and a local penalty achieves a similar result to the one-ring constraint result, with 11.15 times faster performance.

\section{EPIDERMIS MODEL}

The human body is covered by skin, and the epidermis constitutes the outer-most layers of the skin. It is a complex layered structure whose layers (especially the stratum corneum) are much 
stiffer than the underlying hypodermis, fat and muscle tissues. Multilayer skin models have be previously proposed in computer graphics and biomechanics [Flynn and McCormack 2009; Li and Kry 2014; Magnenat-Thalmann et al. 2002]. The layered structure is fundamental to the quality of deformation and formation of wrinkles [Cerda and Mahadevan 2003]. We propose a simple model for the stiffness of the epidermis as an area-preserving potential of the surface of the mesh, which is simply added to the total energy potential to be minimized. Our results show that this simple extension contributes heavily to the appearance of realistic tissue deformation.

For this, we can use the 2D version of the penalty function we formulated in the previous section. The energy density function has the same form as the $3 \mathrm{D}$ version of the penalty, but with a $2 \mathrm{D}$ reduced deformation gradient [Li et al. 2013] $\tilde{\mathbf{F}} \in \mathbb{R}^{3 \times 2}$.

$$
\Psi_{e}\left(\tilde{\mathbf{F}} ; \gamma ; \lambda_{e}\right):=\frac{\lambda_{e}}{12}(\tilde{J}-1)^{2}\left[\gamma(\tilde{J}-1)^{2}+6\right],
$$

where $\tilde{J}=\operatorname{det} \tilde{\mathbf{F}}$, and $\gamma$ controls how steeply severe area change is penalized. In practice, $\gamma=1.0$ seemed to be a good choice in almost all cases we tested for. This energy is defined for the surface triangles of the 3D mesh, and controlled by the first Lamé parameter $\lambda_{e}$, which is defined separately from the volumetric material parameters. For convenience we compute $\lambda_{e}$ from the more familiar Young's modulus $E_{e}$ and Poisson's ratio $v_{e}$ using Equation 3.

We use a high Poisson's Ratio ( $v \in[0.4,0.49])$ for the epidermis energy density functional (16). We then add this energy potential to the original variational problem (1) to find the local minima of elastic potential such that it minimizes surface area change.

To demonstrate the effectiveness of this simple modification, we indent the cylindrical puck example again with the epidermis model as shown in Figure 6. This simulation produces a more organic surface without unnaturally sharp edges.

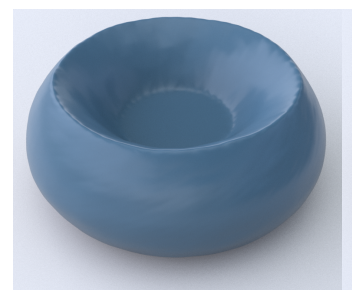

(a) $\mathrm{UNH}, v=0.495$

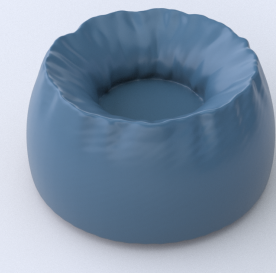

(b) UNH + Epidermis

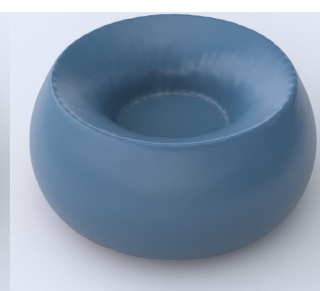

(c) $\mathrm{CNH}, \lambda=40$

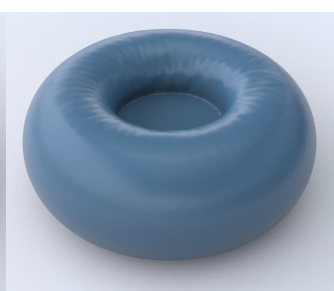

(d) $\mathrm{CNH}+$ Epidermis

Fig. 6. Epidermis Simulation: Cylindrical pucks with unconstrained sides were compressed with Dirichlet boundary conditions in the center. (a) shows the result of the unconstrained neo-Hookean (UNH) model, without the epidermis model, volume constraints, or penalty. (b) shows UNH with just the epidermis energy with $\lambda_{e}=40$ applied to (a); the surface deformation becomes more regular and wrinkles appear as a result (c) and (d) show the corresponding results with our constrained neo-Hookean (CNH) model; (c) shows the effect of just volume preservation and compression penalty while (d) shows the effect of adding the epidermis energy to (c).

\section{RESULTS}

The following results demonstrate the versatility of our method. We use a tetrahedral mesh discretization for all our simulations. Our implementation relied on the Ipopt non-linear optimization package [Wächter and Biegler 2006] to solve the constrained optimization problem proposed in Equation 8. In our experiments, we found that excessive parameter tuning was not required to use 


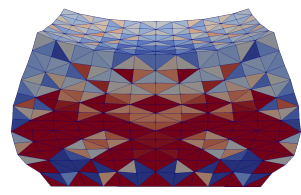

(a) $\mathrm{UNH}, v=0.495$

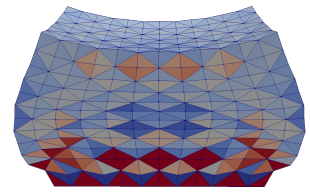

(b) One-ring

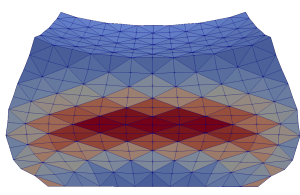

(c) $\mathrm{CNH}, \lambda=100, \beta=1$

Fig. 7. Pressure Distribution: A cube with $12^{3}$ vertices is supported under gravity with the bottom surface fixed with Dirichlet boundary conditions, visualized with the per-tet pressure distribution in the inner body. (a) shows the per-tet Poisson's ratio approach with $v=0.495$, where the effects of volumetric locking results in highly irregular pressure distribution. (b) is the result where a one-ring volume constraint is used, where a checkerboard pattern appears in the pressure distribution due to a lack of stability. Finally, (c) is our method with $\lambda=100, \beta=1$, where the pressure distribution is regular and realistic.

our method with Ipopt: only when used with additional nonlinear constraints, we occasionally tuned the nlp_scaling_max_gradient parameter. For all our examples, we set the Young's Modulus to $\mu=16.0 \mathrm{KPa}$, and we started with $\beta=1.0$ and tuned it to improve performance. However, for just resolving inversions and numerical instability, $\beta=1.0$ works well for all of these examples.

\subsection{Two Tetrahedra}

As the simplest proof of concept example for the volume constraint, we constructed a mesh with two tetrahedra with equal volume joined together by a face as shown in Figure 2. We then compressed one of the tetrahedra with a Dirichlet boundary condition to a plane, to see the effects of the volume constraint. The energy model used is neo-Hookean with $v=0.495$.

As expected, the example without the global volume constraint loses around $50 \%$ of the volume, since the deformation of the compressed tetrahedron only affects its neighbor due to the change in shape of their shared face. When a volume constraint is applied, the unconstrained tetrahedron inflates to twice the original size, keeping the total volume constant.

\subsection{Pressure Distribution}

Following a classic volumetric locking example, we test our methods with a standing cube simulation. A neo-Hookean cube with shear modulus $\mu=10.0$ is supported under gravity with the bottom surface fixed with Dirichlet boundary conditions, results shown in Figure 7. This example demonstrates the effects of external force on the pressure distribution on the body surface and interior with regards to different approaches of simulating incompressibility. Irregular pressure distribution may not visually affect the results much, but when simulating frictional contact or fracture, they might result in unrealistic solutions. The high Poisson's ratio $(v=0.495)$ approach results in obvious irregularities in the pressure distribution, a manifestation of volumetric locking. Per-tet hydrostatic pressures in this case are computed as the the volumetric components of the stress tensors, that is, the negative divergence of the Cauchy stress. The high bulk modulus per each element causes pressure computation from displacement variables to be unreliable. The one-ring volume constraint approach [Irving et al. 2007], which is equivalent to the Average Nodal Pressure element [Bonet and Burton 1998], shows a more regular pressure distribution, but also shows checkerboard patterns. In this case, the pressures are the Lagrange multipliers for the volume constraints, scaled to be in the same units as the volumetric stress then mapped back to the cells. The checkerboard pattern is an artifact of the instability of the one-ring constraint approach, where the averaging of the pressure variables on the nodes allows solutions with such checkerboarding 


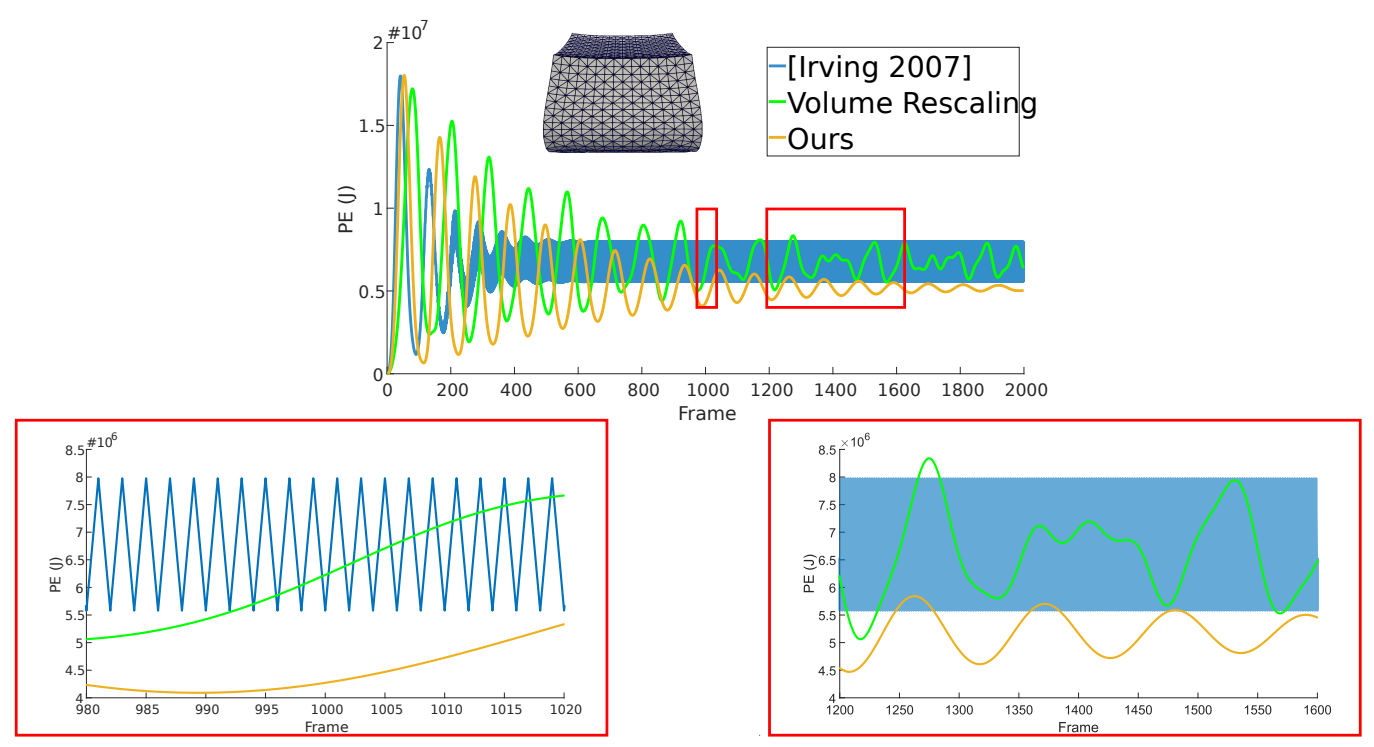

Fig. 8. Dynamic Stability: Energy plot from the simulation of a soft $16^{3}$ cell cube subjected to gravity, with a moderately large timestep of $8 \mathrm{~ms}$. We compare three different algorithms for simulating incompressibility, where the blue curve is the energy plot for the one-ring pressure algorithm of [Irving et al. 2007], the green is a naïve global volume rescaling algorithm, and yellow is ours. [Irving et al. 2007] (blue) shows severe oscillation even with an aggressive clamping of the volume recovery. Note how due to the extreme oscillations in potential energy, the energy plot for the method appears as a thick line in the top plot. The bottom plots show a magnified plot to demonstrate how the energy behaves in small timeframes. The volume rescaling algorithm (green) gains and loses energy arbitrarily due to a unrealistic simulation of the volumetric stress, which can be seen from the irregularities in the plot. Our method (yellow) displays realistic and stable energy behavior, and converges to a stable configuration.

to occur. With our method with one global zone and a local compression penalty of $\lambda=100, \beta=1$, we compute the per-tet pressure as a sum of the average zonal pressure (the Lagrange multiplier of the zonal volume constraint) and the fine-scale pressures computed from the volumetric stress component from the penalty. The bulk modulus is much smaller, compared to $v=0.495$ (corresponding to $\lambda=400$ ) in the Poisson's ratio approach. Therefore volumetric locking is avoided and the volumetric stress components are more regular. Since the volume preserving zone is global and the average pressure is constant throughout the zone, the checkerboard artifact in the Lagrange multipliers is eliminated.

\subsection{Dynamic Stability}

We test the stability of our method in dynamic simulations compared to [Irving et al. 2007] and a naïve volume rescaling method that readers may be tempted to use. We simulate a cube consisting of $16^{3}$ cells with $\mu=100.0$, under gravity. We tested the simulation with a timestep of $8 \mathrm{~ms}$ for a total of 1000 frames. We found that the volume projection in the Irving algorithm can be unstable when used with large timesteps, and clamping of volume preservation forces must be applied to make it stable. However, we found that the clamping threshold to make this specific example not blow up was quite low, resulting in a volume error of $2.4 \%$ at worst. Even with such aggressive clamping, we noticed visible oscillations on the top surface of the cube. We also tested a simple volume rescaling algorithm, where the mesh was projected at every time step based on its center of mass, such 

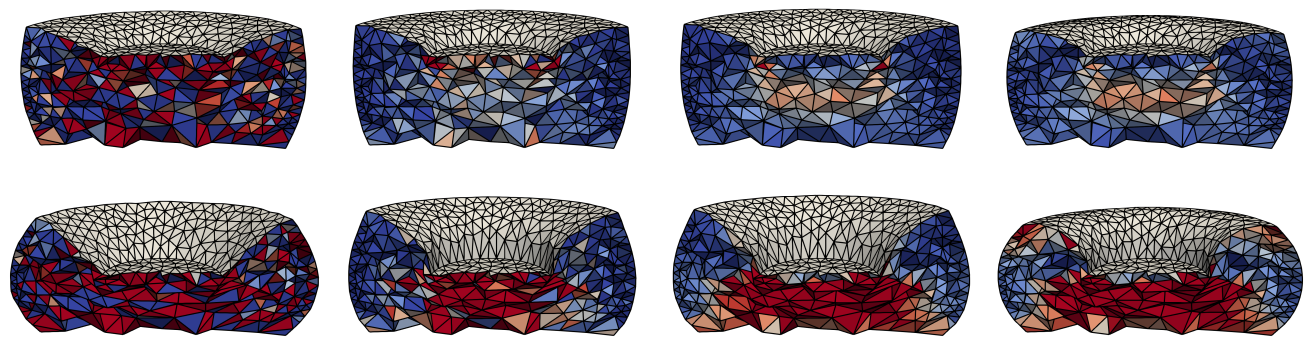

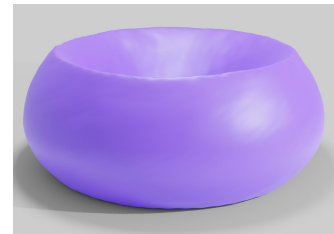

(a) UNH, $v=0.495$

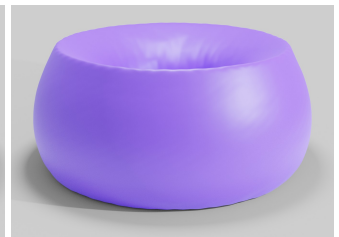

(b) UNH, v $=0.4545$

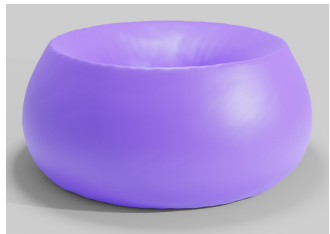

(c) $\mathrm{CNH}$

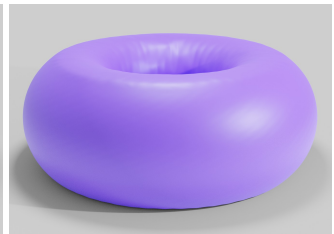

(d) $\mathrm{CNH}+$ Epidermis

Fig. 9. Bulge Test: A cross-section of a simulated puck is shown. The puck is indented at $25 \%$ and $50 \%$ of its height. (a) is the result without volume constraint and $v=0.495$. These base results show visible volume loss of $0.6 \%$ and $1.8 \%$ and artificial stiffness from locking, resulting in no visible bulging at the cylinder top. Also, the pressure distribution visualized by the color scheme shows severe irregularities. (b) is the simulation with $v=0.4545$, which loses $2.1 \%$ and $5.1 \%$ of the total volume respectively. (c) shows the result with volume constraint and $\lambda=100$ and $\beta=9$, showing a realistic bulging due to successful volume preservation. (d) shows the result where an epidermis of $\lambda=100$ was added, where the surface demonstrates a more organic deformation due to surface area preservation.

that the global volume is conserved. We found that this method is highly unstable and virtually impossible with Semi-Implicit Euler integration (where only one Newton step is used) without increasing Poisson Ratio to greater than 0.495, which leads to locking. With full Backward Euler integration, we could get the simulation to not blow up, but it still required a quite high Poisson Ratio of 0.48 . However, an unrealistically exaggerated oscillation of the entire mesh was present even until the final frames. Compared to the other methods, we found our method to be very stable, and the runtimes were comparable (around $2 \%$ faster) to Irving's, which is a semi-implicit method where ours is fully implicit. With our method, this simulation is stable at much higher timesteps, i.e. $33 \mathrm{~ms}$.

The plot of the potential energy for this simulation can be seen Figure 8. Note the extreme oscillations present in the potential energy plot for the one-ring nodal pressure algorithm [Irving et al. 2007] and the unrealistic irregularities in the plot for the volume rescaling algorithm. For a visual comparison of the instabilities of the other two methods compared to ours, please refer to our accompanied video.

\subsection{Bulge Test}

A natural example of an incompressible material is human soft tissue, so to test the effects of our method, we test on the "skin puck" model from [Pai et al. 2018], where the vertices on the bottom are fixed with Dirichlet boundary conditions. When simulating biological tissue, bulging is a crucial visual characteristic that depicts the incompressibility of the underlying material. Therefore, it is important that this simulation shows visually significant bulging under compression. We use a $22 \mathrm{~K}$ tetrahedron simulation mesh for the puck. To produce substantial compression, we animate a set 
of vertices on the top of the puck with a Dirichlet boundary condition moving these vertices down by a fixed amount per time step. We performed a quasi-static simulation where at each step, the animated surface is indented by $1 \%$ of the height. We test the displacement until $50 \%$ of the total height of the puck, which produces extreme compression.

Without the volume constraint, a low Poisson's Ratio $v \in[0.0,0.45]$ will result in little noticeable bulging at the top surface due to volume loss, and a higher $v$ will result in unnaturally stiff visual results and irregular pressure distribution due to volumetric locking. Also, even at $v=0.495$, there was a $1.8 \%$ volume loss at an indentation of $50 \%$ of the puck height.

Adding the volume constraint allows a completely incompressible simulation with realistic pressure solutions for this example, without being a heavy burden on performance in most cases. At $50 \%$ indentation, the amount of volume loss can be made arbitrarily low ${ }^{1}$ with the global volume constraint using any type of energy model and parameters. By contrast, a standard simulation without the constraint produces approximately $22 \%$ volume loss with $v=0.4,5.1 \%$ loss with $v=0.45$, and $1.8 \%$ loss with $v=0.495$. However, without any local compression penalty the simulation converges to an infeasible state with many inverted tets around the border of the Dirichet boundaries.

Adding our local penalty term with $\lambda=100$ allows the simulation to be completely free of inverted tets. Although even with $\beta=0$ the solution does not converge to an infeasible state, the lack of a sufficient resistance to volumetric deformation causes numerical instability and results in a very slow convergence of the nonlinear optimizer during the timesteps with more extreme deformations (after the $25 \%$ indentation). By using $\beta=9$ we were able to achieve better numerical stability, resulting in a $14.84 \%$ faster runtime on total, and $21.17 \%$ faster runtime when only considering the frames after the $25 \%$ indentation where the moving Dirichlet boundary starts to invert elements Finally, with an epidermis model of $\lambda_{e}=100, \beta_{e}=1$ added, we are able to generate a more regular surface deformation and achieve an visually organic deformation overall.

\subsection{Dynamic Impact}

We tested a simple dynamic result of a soft ball consisting of $64 \mathrm{~K}$ tets dropped on the ground (Figure 10). We used a timestep of $1 \mathrm{~ms}$ and $\mu=16.0 \mathrm{KPa}$. Using a per-tet Poisson's ratio $v=0.495$ results in the sphere behaving much stiffer than what the material parameters would suggest, while still losing up to $12.7 \%$ of its volume. When using a per-tet Poisson's ratio of $v=0.45$, the ball retains its appearance of soft elastic deformation, but loses up to $51 \%$ of its original volume. Using our method, we are able to simulate the soft elastic deformations while preserving the volume down to solver accuracy, while being $5.7 \%$ faster than the high Poisson's ratio case and only $3.7 \%$ slower than the $v=0.45$ case.

\subsection{Resolution Consistency}

An important advantage of enforcing volume preservation with zonal constraints is that it allows a way of simulating incompressible objects using a much coarser mesh than by using a traditional 1-field method. Céa's Lemma already couples the quasi-best approximation error with mesh resolution, and since a 1-field FE solutions also couple the bulk modulus to the upper bound of the approximation error, it makes it even harder to use a coarser mesh when bulk modulus is high. However, when incompressibility is decoupled from the bulk modulus, and we can use much smaller $\lambda$, we are able to achieve simulation results of a fine-mesh simulation that is consistent with a much coarser mesh.

${ }^{1} \mathrm{Up}$ to machine precision. 
(a) $\mathrm{UNH}, v=0.45$

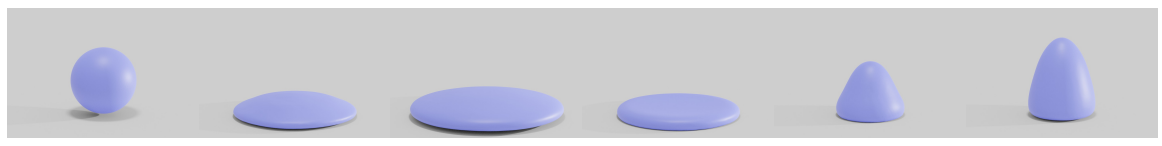

(b) $\mathrm{UNH}, v=0.495$

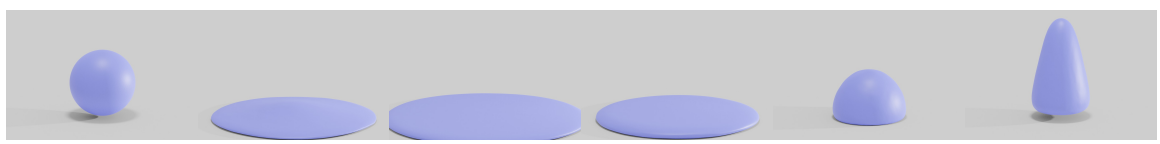

(c) Ours

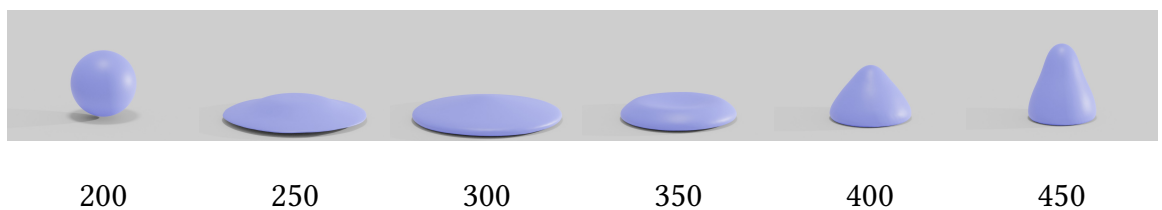

Frame

200

250

300

350

400

Fig. 10. Ball Drop: An elastic sphere consisting of $64 \mathrm{~K}$ tets is dropped to the ground. (a) shows the result with the standard UNH model with per-tet Poisson's ratio $v=0.45$, where the ball loses more than half its volume in the second column. (b) is the UNH result with $v=0.495$, where the volumetric locking makes the ball appear unnaturally stiff. Notice how the ball always retains its spherical shape and just gets flattened and stretched in the vertical direction. (c) is the result using our $\mathrm{CNH}$ model with global volume constraint and local compression penalty with $\lambda$ equivalent to $v=0.45$. Note that the volume of the sphere is preserved, producing a nice "squash-and-stretch" effect, and the artificial stiffness is removed.

(a) UNH, $v=0.45$

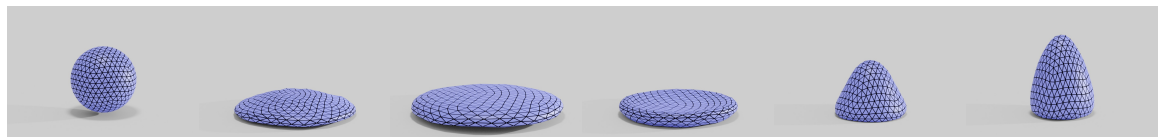

(b) $\mathrm{UNH}, v=0.495$

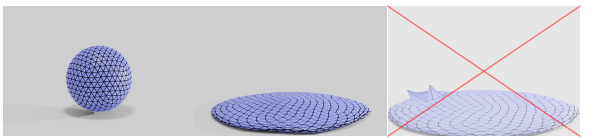

Simulation failed at the 275th frame.

(c) Ours

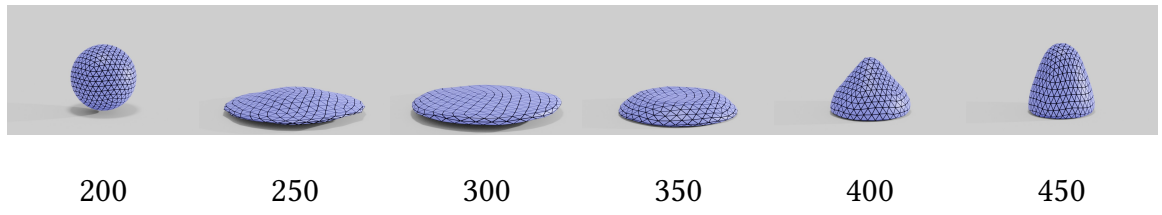

Frame

Fig. 11. Coarse ball: Similar to Figure 10, a much coarser sphere with $4.7 \mathrm{~K}$ tets is dropped to the ground. (a)
shows the UNH result with $v=0.45$, where the ball has lost $52 \%$ of its original volume at the 250 th Frame, but gain $36 \%$ volume at the 300th frame. (b) is the result of using UNH with $v=0.495$, where due to using a coarser mesh the issue of locking is exacerbated, and the simulation fails to converge at the 275th frame. (c) is our result, where the simulation is stable and consistent with the result when using a much finer mesh, demonstrating our advantage of resolution consistency.

When a coarser mesh (4.7K tets) is used, the advantage of our method becomes even clearer (see Figure 11). For the low Poisson's ratio example, the maximum volume loss is almost equal to when using a finer mesh (52.8\%). But after its impact with the ground, the ball actually gains volume due to the severe volumetric deformations resulting in extremely high volumetric elastic force, and the ball gains up to $36.1 \%$ of its initial volume. The high Poisson's ratio case fails to converge after 


\begin{tabular}{|c|c|c|c|c|c|c|c|}
\hline Example & Model & $\mathrm{VC}$ & $\lambda$ & $\beta$ & $\lambda_{e}$ & $t_{r}$ & $i_{\text {avg }}$ \\
\hline Cloth-Body & $\mathrm{SNH}$ & no & 400.0 & & 0.0 & 12.10 & 21.32 \\
\hline Cloth-Body & SNH & no & 40.0 & & 0.0 & 12.93 & 23.09 \\
\hline Cloth-Body & Ours & yes & 120.0 & 6.0 & 0.0 & 12.82 & 17.98 \\
\hline Cloth-Body & Ours & yes & 120.0 & 6.0 & 100.0 & 14.63 & 19.18 \\
\hline Puck & $\mathrm{SNH}$ & no & 40.0 & & 0.0 & 1.55 & 3.00 \\
\hline Puck & SNH & no & 400.0 & & 0.0 & 1.58 & 3.14 \\
\hline Puck & Ours & yes & 100.0 & 0.0 & 0.0 & 2.25 & 4.00 \\
\hline Puck & Ours & yes & 100.0 & 9.0 & 0.0 & 1.93 & 3.38 \\
\hline Puck & Ours & yes & 100.0 & 9.0 & 100.0 & 2.25 & 3.51 \\
\hline Suspend & $\mathrm{SNH}$ & no & 400.0 & & 0.0 & 1.02 & 7.28 \\
\hline Suspend & Ours & yes & 40.0 & 0.0 & & 1.02 & 6.52 \\
\hline Ball (Fine) & $\mathrm{NH}$ & no & 120.0 & & 0.0 & 17.27 & 14.64 \\
\hline Ball (Fine) & $\mathrm{NH}$ & no & 400.0 & & 0.0 & 19.00 & 15.90 \\
\hline Ball (Fine) & Ours & yes & 120.0 & 1.0 & 0.0 & 17.91 & 12.29 \\
\hline Ball (Coarse) & $\mathrm{NH}$ & no & 120.0 & & 0.0 & 1.81 & 15.08 \\
\hline Ball (Coarse) & $\mathrm{NH}$ & no & 400.0 & & 0.0 & 2.50 & 21.84 \\
\hline Ball (Coarse) & Ours & yes & 120.0 & 1.0 & 0.0 & 2.71 & 22.10 \\
\hline Armadillo & SNH & no & 400.0 & & 40.0 & 6.26 & 11.29 \\
\hline Armadillo & Ours & yes & 60.0 & 12.0 & 40.0 & 6.97 & 10.84 \\
\hline Stretch & $\mathrm{SNH}$ & no & 40.0 & & 0.0 & 2.80 & 4.51 \\
\hline Stretch & $\mathrm{SNH}$ & no & 400.0 & & 0.0 & 9.91 & 14.33 \\
\hline Stretch & Ours & yes & 25.0 & 0.0 & 0.0 & 7.89 & 9.92 \\
\hline Stretch & Ours & yes & 25.0 & 1.0 & 0.0 & 7.28 & 9.24 \\
\hline Stretch & Ours & yes & 25.0 & 1.0 & 10.0 & 6.25 & 7.33 \\
\hline Twist & $\mathrm{SNH}$ & no & 100.0 & & 0.0 & 86.06 & 138.33 \\
\hline Twist & Ours & no & 100.0 & 9.0 & 0.0 & 37.32 & 50.33 \\
\hline Twist & Ours & yes & 100.0 & 6.0 & 0.0 & 62.15 & 90.33 \\
\hline Twist & Ours & yes & 100.0 & 6.0 & 15.0 & 103.10 & 92 \\
\hline
\end{tabular}

Table 1. Performance Analysis: Performance table representing average run times per frame, and average Ipopt iterations per frame for major examples. SNH is the Stable neo-Hookean [Smith et al. 2018]. (VC: Volume Constraint, $t_{r}$ : Run Times per Frame (sec.), $i_{\text {avg }}$ : Avg. Ipopt Iterations.)

the 275th frame (corresponds to the 0.275 th second). This failure to converge when using a coarse mesh demonstrates how locking is aggravated when the simulation mesh is coarser, leading to a extremely high approximation error as predicted by Céa's Lemma. However, using our method allows a simulation of a completely volume preserving soft elastic ball even with a very coarse mesh. The visual result is consistent with the result using finer resolution, demonstrating that our method allows a resolution-consistent simulation of volume preserving soft objects.

\subsection{Additional Results}

In addition to the examples above, we show how our method performs in large scale deformations and further demonstrate its robustness. We present three large scale simulations of soft tissue with skin, one is shown in Figure 1, and two examples show a deformation of an armadillo model when pressed between cylinders. To demonstrate robustness. we stretch and twist an elastic cube producing large deformations, which can pose problems for traditional simulators. 


\section{LIMITATIONS AND CONCLUSION}

Although our method generates more realistic volume preservation and reduces locking, for simulations without large deformations, the standard Neo-Hookean model may be sufficient due to its simplicity. This is especially true when no other constraints, such as due to external contact, are present in the simulation. Constrained optimization adds some complexity to the simulation, though our results in Table 1 show that the increase is computation times is not prohibitive in most cases. Overall, our findings demonstrate an inexpensive extension to existing FEM systems can effectively solve the problem of volumetric locking while simulating incompressible materials such as the human body.

Performance. Our formulation uses exact non-linear volume constraints on a non-linear optimization problem to preserve volume exactly in demanding applications like statics and dynamics with large time steps. This limits the choice of optimization solvers to ones that support non-linear equality constraints (e.g., Interior Point or SQP solvers). However, for dynamics problems with smaller time steps, or in applications with tolerance for volume loss/gain, linearizing the volume constraint can drastically simplify the problem. This can reduce the overhead of enforcing equality constraints, while still avoiding locking.

Choosing $\lambda$. By decoupling $\lambda$ from its interpretation as a material parameter, our formulation is faced with an additional challenge, which is to determine how exactly $\lambda$ affects the outcome of a simulation. Fortunately, this is not a significant drawback since material parameters for standard Neo-Hookean FEM simulations also deviate from their measured values due to numerical stiffenning. This means that even the parameters of standard models require manual tuning to reproduce real phenomena in simulation. Luckily data-driven methods for determining simulation parameters (which has seen significant attention in recent literature) are generally agnostic to the true physical meaning of these parameters, and thus are equally as compatible with our method.

In conclusion, we presented a general method for realistic volumetric FEM simulations of human soft tissue. Our method provides exact volume preservation without the artificial stiffness due to volumetric locking using zonal volume constraints. This method gives modelers the ability to define volume preserving zones that conform to anatomical compartments and automatically produces "squash-and-stretch" effects. In addition, we introduced an epidermis model for simulating skin mechanics, as an additional surface area-preserving potential. We also proposed a modification to the energy potential to provide control over local volume flow that results in improved recovery during extreme compression and inversion. We also demonstrated how our method allows stable simulations of volume preserving materials with coarse meshes that are consistent with highresolution simulations. Our approach can be applied to a variety of energy models. In particular, we have demonstrated the effectiveness of these simple modifications to the invariant-based non-linear hyperelastic energies such as the Neo-Hookean and Stable Neo-Hookean energy models.

\section{ACKNOWLEDGEMENTS}

This research was supported in part NSERC, the Canada Research Chairs program, and an NSERC Idea-to-Innovation project co-sponsored by Vital Mechanics. The authors would like to thank Ye Fan for his early contributions to this research. 


\section{REFERENCES}

Alexis Angelidis, Marie-Paule Cani, Geoff Wyvill, and Scott King. 2004. Swirling-sweepers: constant-volume modeling. In 12th Pacific Conference on Computer Graphics and Applications, 2004. PG 2004. Proceedings. 10-15.

Douglas N. Arnold, Franco Brezzi, and Michel Fortin. 1984. A stable finite element for the Stokes equations. Calcolo 21, 4 (1984), 337-344.

Ilya Baran and Jovan Popović. 2007. Automatic rigging and animation of 3d characters. In ACM Transactions on graphics (TOG), Vol. 26. ACM, 72.

Klaus-Jürgen Bathe. 2001. The inf-sup condition and its evaluation for mixed finite element methods. Computers \& structures 79, 2 (2001), 243-252.

Klaus-Jürgen Bathe. 2006. Finite Element Procedures. Prentice Hall. https://books.google.ca/books?id=rWvefGICfO8C

Javier Bonet and A.J. Burton. 1998. A simple average nodal pressure tetrahedral element for incompressible and nearly incompressible dynamic explicit applications. Communications in Numerical Methods in Engineering 14, 5 (1998), 437-449.

Javier Bonet and Richard D. Wood. 2008. 7. Bonet, R. D. Wood, Nonlinear Continuum Mechanics for Finite Element Analysis, Cambridge University Press, Cambridge, UK. Vol. 24. https://doi.org/10.1017/CBO9780511755446

Dietrich Braess. 2007. Finite elements: Theory, fast solvers, and applications in solid mechanics. Cambridge University Press.

Enrique Cerda and Lakshminarayanan Mahadevan. 2003. Geometry and physics of wrinkling. Physical review letters 90,7 (2003), 074302.

Oscar Civit-Flores and Antonio Susín. 2014. Robust treatment of degenerate elements in interactive corotational fem simulations. In Computer Graphics Forum, Vol. 33. Wiley Online Library, 298-309.

Eduardo Alberto de Souza Neto, Francisco M. Andrade Pires, and D.R.J. Owen. 2005. F-bar-based linear triangles and tetrahedra for finite strain analysis of nearly incompressible solids. Part I: formulation and benchmarking. Internat. $\mathcal{F}$. Numer. Methods Engrg. 62, 3 (2005), 353-383.

Raphael Diziol, Jan Bender, and Daniel Bayer. 2011. Robust Real-Time Deformation of Incompressible Surface Meshes. Proceedings - SCA 2011: ACM SIGGRAPH / Eurographics Symposium on Computer Animation, 237-246.

Cormac Flynn and Brendan AO McCormack. 2009. A three-layer model of skin and its application in simulating wrinkling. Computer methods in biomechanics and biomedical engineering 12, 2 (2009), 125-134.

Mihai Frâncu, Arni Asgeirsson, M. Rønnow, and K. Erleben. 2021. Locking-proof Tetrahedra. ACM Transactions on Graphics 40 (2021), 2.

Yuan-cheng Fung. 2013. Biomechanics: mechanical properties of living tissues. Springer Science \& Business Media.

Stefan Hartmann and Patrizio Neff. 2003. Polyconvexity of generalized polynomial-type hyperelastic strain energy functions for near-incompressibility. International journal of solids and structures 40, 11 (2003), 2767-2791.

Gentaro Hirota, Renee Maheshwari, and Ming C. Lin. 2000. Fast volume-preserving free-form deformation using multi-level optimization. Computer-Aided Design 32, 8 (2000), 499 - 512. https://doi.org/10.1016/S0010-4485(00)00038-5

Min Hong, Sunhwa Jung, Min-Hyung Choi, and Samuel W.J. Welch. 2006. Fast Volume Preservation for a Mass-Spring System. IEEE Computer Graphics and Applications 26, 5 (Sept 2006), 83-91. https://doi.org/10.1109/MCG.2006.104

Geoffrey Irving, Craig Schroeder, and Ronald Fedkiw. 2007. Volume Conserving Finite Element Simulations of Deformable Models. ACM Trans. Graph. 26, 3, Article 13 (July 2007). https://doi.org/10.1145/1276377.1276394

Geoffrey Irving, Joseph Teran, and Ronald Fedkiw. 2004. Invertible Finite Elements for Robust Simulation of Large Deformation. In Proceedings of the 2004 ACM SIGGRAPH/Eurographics Symposium on Computer Animation (SCA '04). Eurographics Association, Goslar Germany, Germany, 131-140. https://doi.org/10.1145/1028523.1028541

Alec Jacobson, Ilya Baran, Ladislav Kavan, Jovan Popović, and Olga Sorkine. 2012. Fast automatic skinning transformations. ACM Transactions on Graphics (TOG) 31, 4 (2012), 77.

Doug L James and Christopher D Twigg. 2005. Skinning mesh animations. In ACM Transactions on Graphics (TOG), Vol. 24. ACM, 399-407.

Peter Kaufmann. 2012. Discontinuous Galerkin FEM in Computer Graphics. Ph.D. Dissertation. ETH Zurich.

Ryo Kikuuwe, Hiroaki Tabuchi, and Motoji Yamamoto. 2009. An edge-based computationally efficient formulation of Saint Venant-Kirchhoff tetrahedral finite elements. ACM Transactions on Graphics (TOG) 28, 1 (2009), 8.

Duo Li, Shinjiro Sueda, Debanga R. Neog, and Dinesh K. Pai. 2013. Thin Skin Elastodynamics. ACM Trans. Graph. (Proc. SIGGRAPH) 32, 4 (July 2013), 49:1-49:9.

Pengbo Li and Paul G. Kry. 2014. Multi-layer Skin Simulation with Adaptive Constraints. In Proceedings of the Seventh International Conference on Motion in Games (MIG '14). ACM, New York, NY, USA, 171-176. https://doi.org/10.1145/ 2668084.2668089

Tiantian Liu, Sofien Bouaziz, and Ladislav Kavan. 2017. Quasi-Newton Methods for Real-Time Simulation of Hyperelastic Materials. ACM Trans. Graph. 36, 3, Article 116a (May 2017). https://doi.org/10.1145/2990496

Andreas Longva, Fabian Löschner, Tassilo Kugelstadt, José Antonio Fernández-Fernández, and Jan Bender. 2020. Higher-order finite elements for embedded simulation. ACM Transactions on Graphics (TOG) 39, 6 (2020), 1-14. 
Ives Macêdo, João Paulo Gois, and Luiz Velho. 2009. Hermite interpolation of implicit surfaces with radial basis functions. In 2009 XXII Brazilian Symposium on Computer Graphics and Image Processing. IEEE, 1-8.

Nadia Magnenat-Thalmann, Prem Kalra, Jean Luc Lévêque, Roland Bazin, Dominique Batisse, and Bernard Querleux. 2002. A computational skin model: fold and wrinkle formation. IEEE Transactions on Information Technology in Biomedicine 6, 4 (2002), 317-323.

Aleka McAdams, Yongning Zhu, Andrew Selle, Mark Empey, Rasmus Tamstorf, Joseph Teran, and Eftychios Sifakis. 2011. Efficient Elasticity for Character Skinning with Contact and Collisions. ACM Trans. Graph. 30, 4, Article 37 (July 2011), 12 pages. https://doi.org/10.1145/2010324.1964932

Melvin Mooney. 1940. A theory of large elastic deformation. Journal of applied physics 11, 9 (1940), 582-592.

Matthias Müller, Julie Dorsey, Leonard McMillan, Robert Jagnow, and Barbara Cutler. 2002. Stable Real-time Deformations. In Proceedings of the 2002 ACM SIGGRAPH/Eurographics Symposium on Computer Animation (SCA '02). ACM, New York, NY, USA, 49-54. https://doi.org/10.1145/545261.545269

Dinesh K. Pai, Austin Rothwell, Pearson Wyder-Hodge, Alistair Wick, Ye Fan, Egor Larionov, Darcy Harrison, Debanga Raj Neog, and Cole Shing. 2018. The Human Touch: Measuring Contact with Real Human Soft Tissues. ACM Trans. Graph. 37, 4, Article 58 (July 2018), 12 pages. https://doi.org/10.1145/3197517.3201296

Emmanuel Promayon, Pierre Baconnier, and Claude Puech. 1996. Physically Based Deformations Constrained in Displacements and Volume. Computer Graphics Forum (Proc. of Eurographics '96) 15 (08 1996). https://doi.org/10.1111/14678659.1530155

Mike A. Puso and Jerome M. Solberg. 2006. A stabilized nodally integrated tetrahedral. Internat. f. Numer. Methods Engrg. 67, 6 (2006), 841-867.

Ronald S. Rivlin and Eric K. Rideal. 1948. Large elastic deformations of isotropic materials IV. further developments of the general theory. Philosophical Transactions of the Royal Society of London. Series A, Mathematical and Physical Sciences 241, 835 (1948), 379-397. https://doi.org/10.1098/rsta.1948.0024 arXiv:https://royalsocietypublishing.org/doi/pdf/10.1098/rsta.1948.0024

Damien Rohmer, Stefanie Hahmann, and Marie-Paule Cani. 2009. Exact Volume Preserving Skinning with Shape Control. In Proceedings of the 2009 ACM SIGGRAPH/Eurographics Symposium on Computer Animation (SCA '09). ACM, New York, NY, USA, 83-92. https://doi.org/10.1145/1599470.1599481

Eftychios Sifakis and Jernej Barbic. 2012. FEM Simulation of 3D Deformable Solids: A Practitioner's Guide to Theory, Discretization and Model Reduction. In ACM SIGGRAPH 2012 Courses (SIGGRAPH '12). ACM, New York, NY, USA, Article 20, 50 pages. https://doi.org/10.1145/2343483.2343501

Breannan Smith, Fernando De Goes, and Theodore Kim. 2018. Stable Neo-Hookean Flesh Simulation. ACM Trans. Graph. 37, 2, Article 12 (March 2018), 15 pages. https://doi.org/10.1145/3180491

Alexey Stomakhin, Russell Howes, Craig Schroeder, and Joseph M. Teran. 2012. Energetically Consistent Invertible Elasticity. In Proceedings of the 11th ACM SIGGRAPH / Eurographics Conference on Computer Animation (EUROSCA'12). Eurographics Association, Aire-la-Ville, Switzerland, Switzerland, 25-32. https://doi.org/10.2312/SCA/SCA12/025-032

Theodore Sussman and Klaus-Jürgen Bathe. 1987. A finite element formulation for nonlinear incompressible elastic and inelastic analysis. Computers \& Structures 26, 1-2 (1987), 357-409.

Joseph Teran, Eftychios Sifakis, Geoffrey Irving, and Ronald Fedkiw. 2005. Robust Quasistatic Finite Elements and Flesh Simulation. In Proceedings of the 2005 ACM SIGGRAPH/Eurographics Symposium on Computer Animation (SCA '05). ACM, New York, NY, USA, 181-190. https://doi.org/10.1145/1073368.1073394

Rodolphe Vaillant, Loïc Barthe, Gaël Guennebaud, Marie-Paule Cani, Damien Rohmer, Brian Wyvill, Olivier Gourmel, and Mathias Paulin. 2013. Implicit skinning: real-time skin deformation with contact modeling. ACM Transactions on Graphics (TOG) 32, 4 (2013), 125.

Wolfram von Funck, Holger Theisel, and Hans-Peter Seidel. 2007. Explicit Control of Vector Field Based Shape Deformations In 15th Pacific Conference on Computer Graphics and Applications (PG'07). 291-300. https://doi.org/10.1109/PG.2007.26

Andreas Wächter and Lorenz T. Biegler. 2006. On the implementation of an interior-point filter line-search algorithm for largescale nonlinear programming. Mathematical Programming 106, 1 (01 Mar 2006), 25-57. https://doi.org/10.1007/s10107004-0559-y

Huamin Wang and Yin Yang. 2016. Descent methods for elastic body simulation on the GPU. ACM Transactions on Graphics (TOG) 35, 6 (2016), 1-10.

Ofir Weber, Olga Sorkine, Yaron Lipman, and Craig Gotsman. 2007. Context-aware skeletal shape deformation. In Computer Graphics Forum, Vol. 26. Wiley Online Library, 265-274.

Holger Wendland. 2004. Scattered data approximation. Vol. 17. Cambridge university press.

Seung-Hyun Yoon and Myung-Soo Kim. 2006. Sweep-based Freeform Deformations. Comput. Graph. Forum 25 (09 2006), 487-496. https://doi.org/10.1111/j.1467-8659.2006.00968.x 